\title{
Performance Evaluation of Limited Fractional Guard Channel for SFR-Based LTE-Advanced Networks
}

\author{
Mahammad A. Safwat ${ }^{1 *}$, Hesham M. El-Badawy ${ }^{1}$, Ahmad Yehya ${ }^{2}$, H. El-Motaafy 3 \\ ${ }^{1}$ Network Planning Department, National Telecommunication Institute, Cairo, Egypt. \\ 2 Department of Electrical Engineering, Al-azhar University, Cairo, Egypt. \\ ${ }^{3}$ Department of Electronics and Computer Engineering, HIT, Egypt. \\ * Corresponding author. Tel.: +201115156446; email: mahammad.safwat@nti.sci.eg \\ Manuscript submitted September 5, 2014; accepted March 25, 2015. \\ doi: 10.17706/ijcce.2015.4.4.264-273
}

\begin{abstract}
Call admission control (CAC) is a key enabling technique to ensure quality of service (QoS) provisioning for mobile users. Limited fractional guard channel is one of most effective strategy in call admission control. Inter-cell interference coordination (ICIC) is considered as the most promising approach for LTE to mitigate inter-cell interference. One important approach for ICIC is soft frequency reuse (SFR). In this paper, a new analytical model is derived to evaluate LFGC strategy in SFR-based systems. The performance measures of interest are new call blocking probability, handover dropping probabilities, throughput and mean time response. Further, discrimination in the analysis between cell-core and cell-edge is performed. All performance metrics is deduced separately at each cell zone.
\end{abstract}

Key words: Queuing, limited fractional guard channel, soft frequency reuse.

\section{Introduction}

Call Admission Control plays an important role in Quality of Service (QoS) provisioning for wireless communication systems [1]. The performance of CAC techniques has a direct impact on both users' individual performance as well as on the overall network performance. Effective use of the limited wireless resources has become more and more important, and considerable efforts have been focused on call admission control (CAC) in order to maintain the required QoS.

The call admission control (CAC) performance in a cellular network is specified by the blocking probability of new calls $\left(P_{B}\right)$ in a cell and dropping probabilities $\left(P_{D}\right)$ of handover calls entering a cell. Since dropping an ongoing call is generally more annoying to a mobile user than blocking a new call request [2], a higher priority is normally assigned in CAC for handover calls over the new ones to minimize the call dropping probability. So handover prioritization schemes result in a decrease of handover call dropping rate and in an increase of new call blocking rate that, in turn, reduces the total admitted traffic. In order to overcome this problem, different policies have been proposed: fractional guard channel (FGC), limited fractional guard channel (LFGC) and the uniform fractional guard channel (UFGC) [2]-[4]. The FGC policy uses a vector $\beta=\left[\beta_{0}, \ldots, \beta_{i . .}, \beta_{N-1}\right]$ to accept the new calls, where $0 \leq \beta_{i} \leq 1$ (for $i=0, \ldots, N-1$ ). This policy accepts new calls with probability $\beta_{i}$ when $i$ channels are busy. LFGC and UFGC schemes are particular examples of FGC scheme. In the Uniform FGCP, the acceptance probability has a constant value $\beta^{*}$ that is independent of number of occupied channels. LFGC scheme controls the QoS by effectively varying the average number of reserved channels by a fraction of one. In LGCP, there are three possible admission 
probabilities for new calls $\left(1, \beta_{l}, 0\right)$ where $\beta_{l}<1$. When the cell state $(i)$ is less than $T$, the admission probability is unity. When the cell state $(i)$ is equal to $T$, the admission probability is $\beta_{l}$; finally when the cell state ( $i$ ) is greater than $T$, the admission probability is 0 , where $T$ is a design parameter [4].

Due to the requirement of high spectrum efficiency, the frequency reuse factor of one is targeted for next generation OFDMA-based networks. Such a frequency planning strategy can lead to unacceptable ICI levels experienced especially by users located at the cell-edge area. Soft frequency reuse (SFR) is considered as an effective frequency reuse scheme to mitigate ICI as well as maintaining spectral efficiency [5]. The Resources Blocks (RBs) (basic resource element in LTE networks) are distributed between mobile users according to SFR policy. In SFR, for each cell in the network; the cell is divided into two parts: cell-edge and cell-core. Cell-edge users are confined to cell-edge RBs while cell-core users can be access to the cell-core RBs and can also be access to the cell-edge RBs but with less priority than cell-edge users [5], [6].

Most research on SFR concerns performance metrics due to spectral efficiency and average Signal to Interference plus Noise Ratio (SINR). Studying the performance metrics of the system due to resources availability by CAC methods is rarely covered in most research although the shortage of system resources is an essential problem that ICI result. In [7]-[9], a call admission control for SFR is provided using two dimensions Markov chain. A Queue model for SFR scheme is deduced in [7] and expressions for blocking and outage probability is obtained. In [8] the call admission control in SFR-based systems is derived analytically. A comparison between the SFR-based systems and non-SFR- based systems is executed. The impact of spectrum handoff, which is between cell-edge and cell-core users, is studied in [9] and is compared with SFR without spectrum handoff. In all these papers, the influence of handover process in cell-edge performance is not addressed although the handover mobile user depends on cell-edge RBs in order not to interfere with adjacent cells RBs. These lead to dramatic increasing in blocking probability at cell-edge. In [10], the handover impact is considered using UFGC scheme which provides priority for handover calls by throttle the acceptance of new call.

In this work, a LFGC is used in QoS provisioning in presence of SFR-ICI mitigation scheme. This scheme is distinguished from UFGC scheme in that it combines the guard channel schemes with the acceptance probability ones. So it provides better performance with hard constraint in QoS requirements [2]. Two dimensions Markov chains are used for modeling with discrimination between core and edge mobile calls. The deduced performance metrics is separately calculated at cell-edge and cell-core in order to capture the actual performance at each cell zone. An expression for blocking and dropping probabilities, throughput and mean time response is deduced. To over helm the complexity of the proposed model, the Successive over Relaxation (SOR) iterative method is used to solve Markov process. The model under investigation is irreversible Markov process. So, the SOR may be one of the most suited techniques to obtain steady state probabilities and the required performance metrics. SOR not only supports the feasible solution but also, it gives sufficient stability for the obtained results.

This paper is organized as follows: In Section 2, the system model for SFR with LFGC is presented. The Queuing analysis is introduced in Section 3, Section 4 presents system performance metrics, Numerical results and analysis is provided in Section 5. Finally, conclusion is presented in Section 6.

\section{System Model}

A homogeneous multi-cellular system is assumed that has the same traffic patterns. This allows considering only one cell for performance study and all other cell catch the interaction through handoff call arrival process. The cell is divided into edge and core according to soft frequency reuse scheme used.

In this model the following assumptions are considered and in consistence with previously published work in [7]. So it may be summarized in the following points: 
- The basic resource element considered in this paper is the physical resource block (RB) which spans both frequency and time dimensions. The component frequencies of one RB can be either contiguous or disjoint. The time duration of the RB is defined by one transmission time interval (TTI). A RB can be assigned to only one user at a time.

- $N$ is the number of available RBs that can be used for transmission in each TTI in the cell. The maximum number of RBs that can be assigned to the edge-users and core-users is $E$ and $C$ respectively; the ratio of cell-edge RBs to the total number of RBs in each cell is $\eta$, so $E=\eta N$ where $E+C=N$.

- Let $G_{e}$ be the percent of cell-edge RB reserved for guard channel, and $E_{h}$ is the RB assigned to handover users and $E_{e}$ is resident RBs which can be used by new call and handover users, so $E_{h}=G_{e} E$ and $E=E_{e}+E_{h}$, also let $N_{n}$ the total number of RB assigned for new call users in the whole cell so we have $N_{n}=$ $C+E_{e}$.

- Users are uniformly distributed in a cell. A new call follows a passion process with the mean arrive rate $\lambda$ calls $\backslash$ sec. Users are divided into cell edge users and cell core users by SINR. The distance between users to LTE-Advanced eNodeB in a cell is the only determining factor to SINR. Let $\lambda_{c}$, $\lambda_{e}$, denote the arrival rate for new calls in cell-core, cell-edge respectively. So $\lambda=\lambda_{c}+\lambda_{e,}$. $\lambda_{h}$ is the arrival rate for handover calls.

- The cell-edge RB is available for both of cell-edge users (when $i<T$ ) and handover users and if there are none of them, it can be occupied by cell-core users.

- A cell-edge user may be blocked if all cell-edge RBs in the cell is occupied by cell-edge users or handover users. A cell-core user may be blocked if there is no more cell-core RBs or cell-edge RBs in target cell.

- The cell-edge user can access only one radio resource of guard channels with probability $\beta_{l}$ if all cell-edge RBs are occupied.

- An ongoing handover call may be dropped if all cell-edge RBs in the target cell is occupied by cell-edge users or handover users.

- System may force the cell-core call which has already connected to the networks to be terminated if the cell-core call has occupied cell-edge RBs and a new cell-edge user initialized a new call simultaneously or an ongoing handover call entered the cell.

- Cell-edge user is served from first RB of guard channel rather than force cell-core user to terminate the ongoing call which is connected to cell-edge RB.

- The call duration time for new and handover call in both cell-edge and cell-core is assumed to be exponentially distributed with mean $\mu^{-1}$ sec.

\section{LFGC/SFR Markov Model}

A two dimension Markov chain is used to model LFGC in collaboration with SFR. The system state is defined as $(i, j)$ with $i$ representing the number of RBs used by cell-core users, $j$ representing the number of RBs used by cell-edge and handover users. Then, a two dimensional state space $\Gamma$ can be defined as:

$$
\Gamma=\{(i, j) \mid 0 \leq i \leq N, 0 \leq j \leq E, i+j \leq N\}
$$

Fig. 1 illustrates the state transition diagram in LFGC/ SFR. Let $\pi(i, j)$ be the steady state probability for a valid state $(i, j) \in \Gamma$.

The steady state probabilities should satisfy the normalization constraint.

$$
\sum_{(i, j) \in \Gamma} \pi(i, j)=1
$$


Based on the state diagram shown in Fig. 1, the set of global balance equations can be deduced: For boundary states:

For the state $(i, j)=(0,0)$

$$
\left(\lambda_{c}+\lambda_{e}+\lambda_{h}\right) \pi(0,0)=\mu(\pi(1,0)+\pi(0,1))
$$

For the state $(i, j)=(0, E)$;

$$
\left(\lambda_{c}+E \mu\right) \times \pi(0, E)=\lambda_{h} \pi(0, E-1)+\mu_{c} \pi(1, E)
$$
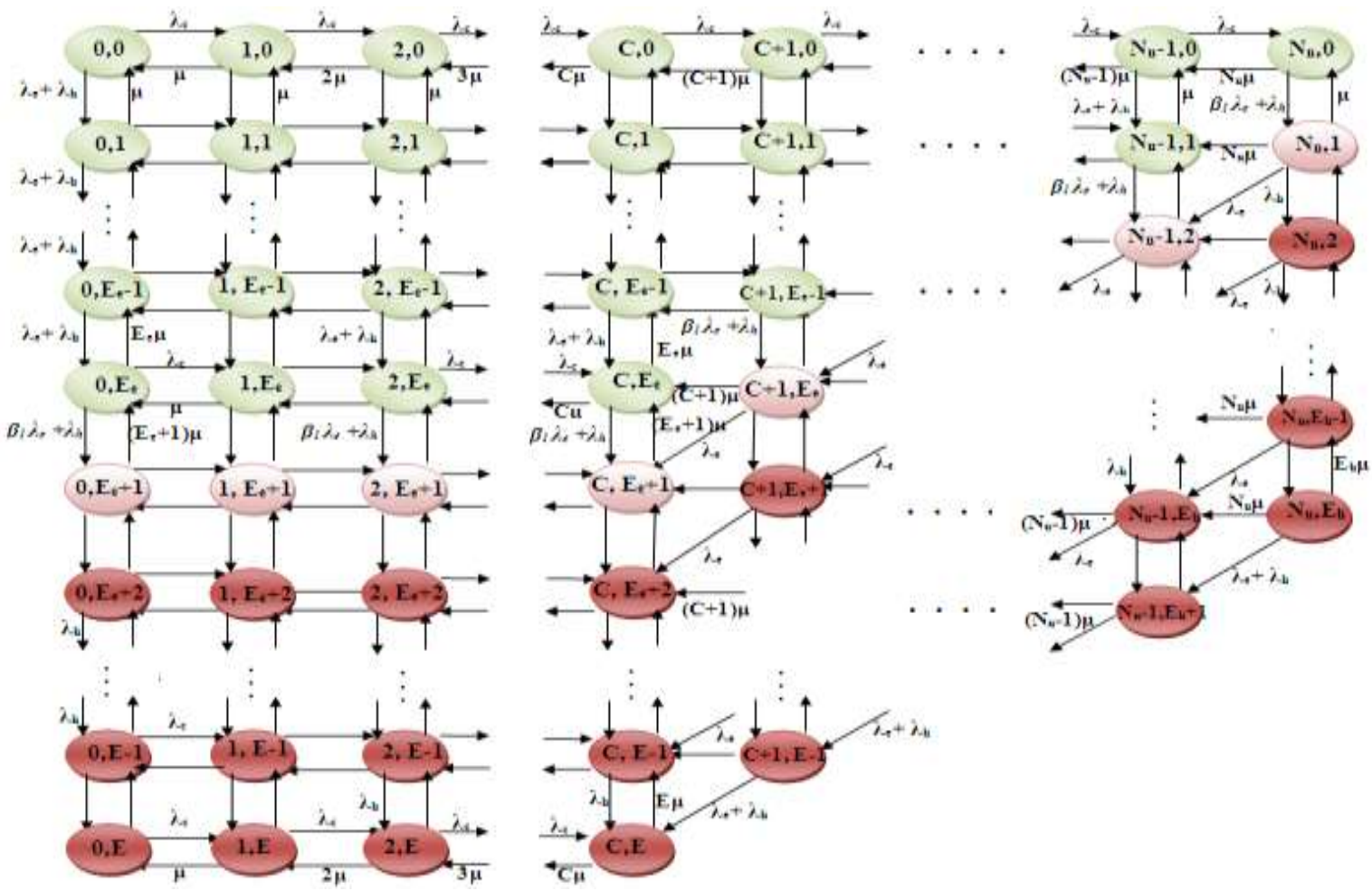

Fig. 1. The state diagram of SFR with LFGC.

For the state $(i, j)=\left(N_{n}, 0\right)$;

$$
\left(\beta_{l} \lambda_{e}+\lambda_{h}+N_{n} \mu\right) \times \pi\left(N_{n}, 0\right)=\lambda_{c} \pi\left(N_{n}-1,0\right)+\mu \pi\left(N_{n}, 1\right)
$$

For the state $(i, j)=(C, E)$;

$$
(C \mu+E \mu) \pi(C, E)=\lambda_{c} \pi(C-1, E)+\lambda_{h} \pi(C, E-1)+\left(\lambda_{e}+\lambda_{h}\right) \pi(C+1, E-1)
$$

For non-boundary states, it can be divided into when $i+j<N_{n}$ and $i+j>N_{n}$ as follows.

First when $i+j<N_{n}$, for states $1 \leq i<N_{n} ; 1 \leq j<E_{e}$

$$
\begin{gathered}
\left(\lambda_{c}+\lambda_{e}+\lambda_{h}+i \mu+j \mu\right) \pi(i, j)=\lambda_{c} \pi(i-1, j)+(i+1) \mu \pi(i+1, j)+\left(\lambda_{e}+\lambda_{h}\right) \pi(i, j-1)+ \\
(j+1) \mu \pi(i, j+1)
\end{gathered}
$$

Second when $i+j>N_{n}$, for states $C<i<N_{n} ; 0<j<E ; i+j<N$ 


$$
\begin{gathered}
\left(\lambda_{e}+\lambda_{h}+i \mu+j \mu\right) \times \pi(i, j)=\lambda_{h} \pi(i, j-1)+(i+1) \mu \pi(i+1, j)+\lambda_{e} \pi(i+1, j-1) \\
+(j+1) \mu \pi(i, j+1)
\end{gathered}
$$

In case of $i+j=N_{n}$

For states $C<i<N_{n}, i+j=N_{n}$;

$$
\begin{gathered}
\left(\beta_{l} \lambda_{e}+\lambda_{h}+i \mu+j \mu\right) \pi(i, j)=\lambda_{c} \pi(i-1, j)+\left(\lambda_{e}+\lambda_{h}\right) \pi(i, j-1)+(j+1) \mu \pi(i, j+1)+ \\
(i+1) \mu \pi(i+1, j)
\end{gathered}
$$

In case of $i+j=N$

For states $C<i<N_{n} ; i+j=N$

$$
\begin{gathered}
\left(\lambda_{e}+\lambda_{h}+i \mu+j \mu\right) \times \pi(i, j)=\lambda_{h} \pi(i, j-1)+(i+1) \mu \pi(i+1, j)+\lambda_{e} \pi(i+1, j-1)+ \\
(j+1) \mu \pi(i, j+1)
\end{gathered}
$$

The balance equations of the remaining states can be deduced in the same manner.

To over helm the complexity of the multi-dimension Markov model, the Successive over Relaxation (SOR) iterative method is used to enhance the solution of Markov process. The SOR is a variant of the Gauss-Seidel method for solving a linear system of equations, resulting in faster convergence.

A set of SOR equations can be deduced from balance equation, the left hand side of these equations is a new value of steady state probability which is obtained iteratively using previous value for steady state probability on the right hand side. The speed of convergence is determined by relaxation factor $\omega$; the choice of relaxation factor is not necessarily easy, and depends upon the properties of the coefficient matrix. For symmetric, positive definite matrices it can be proven that $0<\omega<2$ will lead to convergence, but we are generally interested in faster convergence rather than just convergence.

The steps of SOR algorithm are as follows [7], [10]:

1. The number of total valid states in the set $\Gamma$ is computed;

$$
S=\left(N_{n}+1\right) \times(E+1)-0.5 \times\left(E_{e}+1\right) \times E_{e}
$$

2. For $\forall(i, j) \in \Gamma$, let the initial probability be

$$
\Pi^{(0)}(i, j)=1 / s
$$

3. Let the convergence criteria be $\varepsilon$, the relaxation factor be $w(1 \leqq w<2)$, and the iteration $k=1$;

4. Calculate the SOR equations in sequence.

5. If we have

$$
\begin{gathered}
\Pi^{(k)}(i, j)=\frac{\Pi^{(k)}(i, j)}{\sum_{(i, j)} \Pi^{(k)}(i, j)} \\
\left\|\Pi^{(k)}(i, j)-\Pi^{(k-1)}(i, j)\right\| \leq \varepsilon
\end{gathered}
$$

Then exit to step 6; otherwise let $k=k+1$ and reexecute steps 4 and 5 .

6. Output the steady state probability and calculate the performance metrics. 


\section{Performance Metrics}

The performance of the current model will be evaluated by determining the blocking probability $\left(P_{B}\right)$, the dropping probability $\left(P_{D}\right)$, throughput and mean time response. Let $\psi_{b e}$ and $\psi_{b c}$ be the subsets of states where a new arriving cell-edge and cell-core user are blocked respectively.

$$
\begin{gathered}
\Psi_{b e}=\left\{\pi(i, j) \in \Psi_{b e 1} ; i=E_{e}, 1 \leq j \leq C\right. \\
\cup \pi(i, j) \in \Psi_{b e 2} ; E_{e}<i \leq E, 1 \leq j \leq C \\
\left.\cup \pi(i, j) \in \Psi_{b e 3} ; C \leq i \leq N_{n}, 0 \leq j \leq E_{h}, i+j=N_{n}\right\} \\
\Psi_{b c}=\left\{\pi(i, j) \in \Psi_{b c} ; C \leq i \leq N_{n}, 0 \leq j \leq E_{h}, i+j \geq N_{n}, i+j \leq N\right\}
\end{gathered}
$$

Then the blocking probabilities at cell-edge $P_{B e}$ and at cell-core $P_{B c}$ are calculated as follow:

$$
\begin{gathered}
P_{B e}=\left(1-\beta_{l}\right) \sum_{(i, j) \in \Psi_{b e 1}} \xi_{e} \pi(i, j)+\sum_{(i, j) \in \Psi_{b e 2}} \xi_{e} \pi(i, j)+\left(1-\beta_{l}\right) \sum_{(i, j) \in \Psi_{b e 3}} \xi_{e} \pi(i, j) \\
P_{B c}=\sum_{(i, j) \in \Psi_{b c}} \xi_{c} \pi(i, j)
\end{gathered}
$$

where $\xi_{c}$ is the probability to have users in cell-core and $\xi_{e}$ is the probability to have users in cell-edge.

Finally let $\psi_{d}$ be the subsets of states where the system forces to terminate the ongoing handover call.

$$
\Psi_{d}=\left\{\pi(i, j) \in \Psi_{d} ; i=E, 1 \leq j \leq C\right\}
$$

Then the cell dropping probability is calculated as:

$$
P_{D}=\sum_{(i, j) \in \Psi_{d}} \xi_{e} \pi(i, j)
$$

The throughput $\bar{Y}$ is the weighted average of the service rates where the steady state probabilities serve as weights. Let $\bar{Y}_{e}$ be the mean throughput at cell-edge.

$$
\bar{Y}_{e}=\sum_{i=1}^{N_{n}} \sum_{j=1}^{E} \mu(j) \pi(i, j)
$$

The proposed queuing model has $N$ RBs and $N$ system places, and there no waiting positions or queues for overloaded traffic, so it is called loss system. If there is no RB for incoming call, it is simply blocked. So part of the arrivals are lost and the effective arrival rate at each state is equal to arrival rates at each state minus the loss rates. Let $\widetilde{\lambda_{e}}, \widetilde{\lambda_{c}} \& \widetilde{\lambda_{h}}$ be the effective arrival rates for new (at edge \& core) and handover call respectively:

$$
\begin{gathered}
\widetilde{\lambda_{e}}=\sum_{i=0}^{N_{n}} \sum_{j=0}^{E_{e}-1} \lambda_{e} \pi(i, j)+\sum_{i=0}^{i=N_{n}} \beta_{l} \lambda_{e} \pi\left(i, E_{e}\right) \\
\widetilde{\lambda_{c}}=\sum_{i=N_{n}-C, j=1}^{i=N_{n}-1, j=E_{e}} \lambda \pi(i, j) \\
\widetilde{\lambda_{h}}=\sum_{i=0}^{N_{n}} \sum_{j=0}^{E-1} \lambda_{h} \pi(i, j)
\end{gathered}
$$

Let $\widetilde{\lambda_{t}}$ be the total arrival rate at each state which is equal to the summation of all arrival rates entering each state. From Fig. 1, some states have arrivals from new and handover calls and other with only handover calls.

Using little's law; the mean time response at cell-edge zone is 


$$
W_{e}=\frac{L_{e}}{\lambda_{m e}}
$$

where $L_{e}$ is the average number of served users at cell-edge and $\lambda_{m e}$ is the mean arrival rate at the whole cell-edge. It can be obtained by multiplying arrival rate at each state by its steady state probability which serves as weights.

$$
\lambda_{m e}=\sum_{i=1}^{i=N_{n}} \sum_{j=1}^{j=E} \widetilde{\lambda_{t e}}(i, j) \pi(i, j)
$$

where $\widetilde{\lambda_{t e}}$ is the total arrival rate at each state at cell-edge. The same analysis can be executed at cell-core zone.

\section{Numerical Results and Analysis}

In this section, the behavior of the system is illustrated. The blocking probability $P_{B}$ and dropping probability $P_{D}$ are the metrics under consideration for QoS.

The effect of new and handover arrival rate in the performance metric is investigated. The queuing model parameters for the presented results are as follow: the available RBs in the cell $(N)$ is 48; the ratio of cell-edge RBs to total cell RBs is $1 / 3$; the probability that there are users at the edge of the cell $\xi_{e}$ is $1 / 2$, the mean service period $(\mu)$ is 90 seconds. The SOR parameters are $\omega=1.05$, the convergence condition $\varepsilon=10^{-5}$ and $k=1000$.

Fig. 2 illustrate the blocking probability $P_{B}$ of the system as a function of handover arrival rates with separation between edge and core users when $G_{e}=0.25$ and $\beta_{l}=0.5$. For convince and reasonability, the values of handover arrival rate were chosen to be not to exceed the arrival rate of new call for cell edge user. It can be noticed that the blocking probability increases with increasing of handover rate in both cell-edge and cell-core. This is as a result of having more handover requests, so the system will starve to serve the handover requests in price of blocking more and more new call requests. The effect of $\lambda_{h}$ in blocking probability of cell-edge users is greater than its effect in cell-core ones; this is clear when comparing Fig. 2(a) and Fig. 2(b). This is because the cell-edge RBs is admitted for both cell-edge and handover users and so by having more handover requests, more new call will be blocked. The cell-core RBs is dedicated for cell-core users and are not available for handover users and so $\lambda_{h}$ has limited impact on it as illustrated in Fig. 3 . The blocking of cell-core users is due to the cell-core users can also access the cell edge RBs in SFR policy but with less priority than cell edge users. The obtained results show that the merging of LFGC and SFR models are leading to more inspection of system behavior. The manipulation of LFGC based model or SFR based model can't be used to analyze the contribution ratio of cell-edge and cell-core users. The results of Fig. 2 may be used as an evidence for the stability and sustainability of the proposed model.

Fig. 3 shows the blocking probability at different values of $G_{e}$ as a function of new call arrival rate at cell-edge. The handover arrival rates $\lambda_{h}=0.25$ of new call arrival rates at cell-edge and $\beta_{l}=0.5$. It is observed that as $G_{e}$ increases, the blocking probability significantly increases. This because of the remaining RBs for new calls will decrease and so block more and more new call requests.

Fig. 4 explains the effect of handover arrival rate on dropping probability. The new call acceptance probability $\beta_{l}$ and percentage of guard channels $G_{e}$ are 0.5 and 0.25 respectively. It is clear that the dropping probability increases with increasing of $\lambda_{h}$. This is as a result of having more handover request, so more radio resources are occupied. Then, the probability for serving handover calls is reducing and more handover calls are dropped.

Fig. 5 depicts the mean throughput at cell-edge as a function of handover arrival rates. It can be noticed that the throughput increases with increasing handover arrival rates. This due to increasing in the 
occupation rate of guard channels by handover calls. So the total number of admitted calls at time unit increases (by increasing in handover rates) and consequently more RBs are occupied which improve the throughput.

Fig. 6 illustrates the impact of number of guard channels in mean time response at cell-edge. The guard channels are not available for call admission till all other RBs at cell-edge are occupied. So at light traffic, the number of available RBs is lower than heavy traffic case because of guard channels. Then the mean time response decreases in proportional with the number of guard channels.

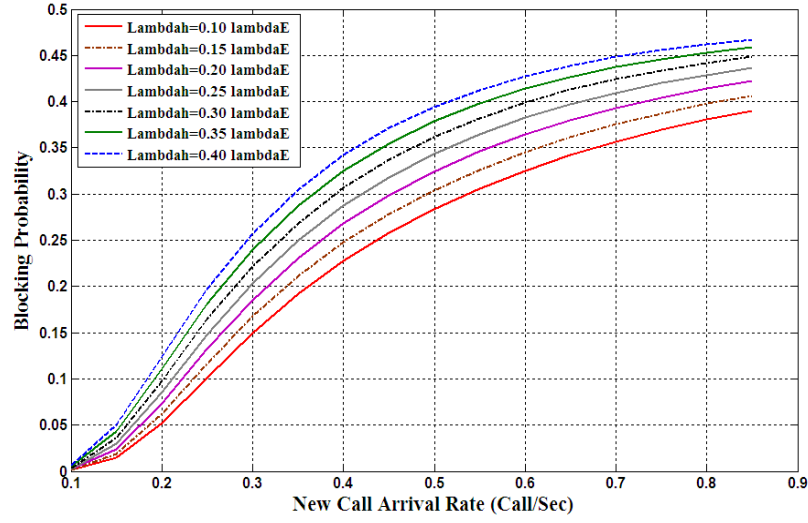

(a)

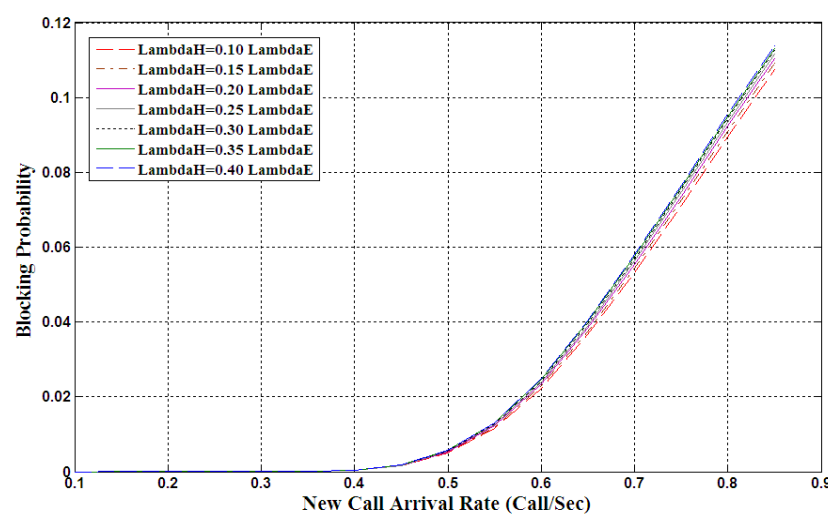

(b)

Fig. 2. Blocking probability of for different $\lambda_{h}, N=48, E=16, E_{e}=12$ at (a) edge part (b) core part.

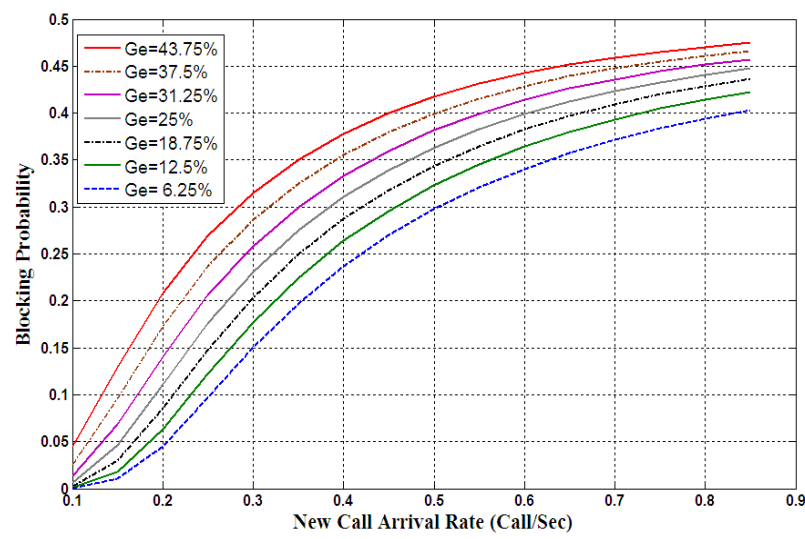

Fig. 3. Blocking probability for cell-edge user as a function of $G_{e}, N=48, E=16, \lambda_{h}=0.25 \lambda_{e}$ Call $\backslash \mathrm{Sec}$.

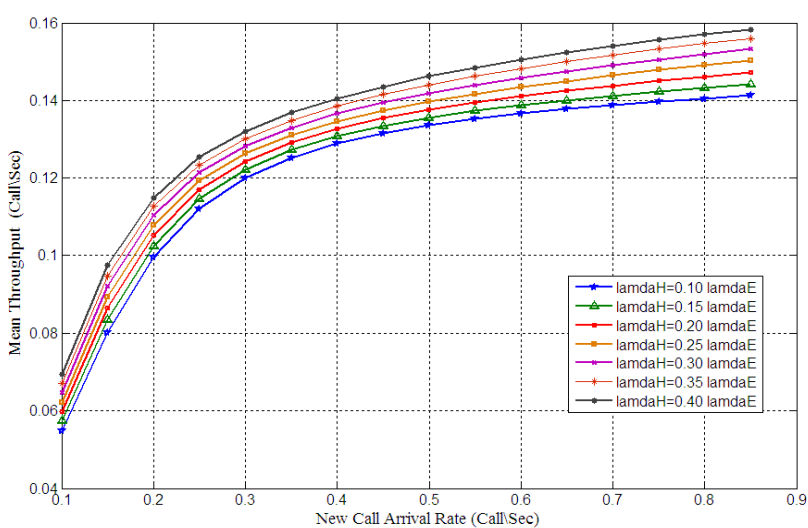

Fig. 5. Mean throughput with different $\lambda_{h}$ at cell-edge, $N=48, E=16, E_{e}=12, \mu=1 / 90$ Sec- 1 .

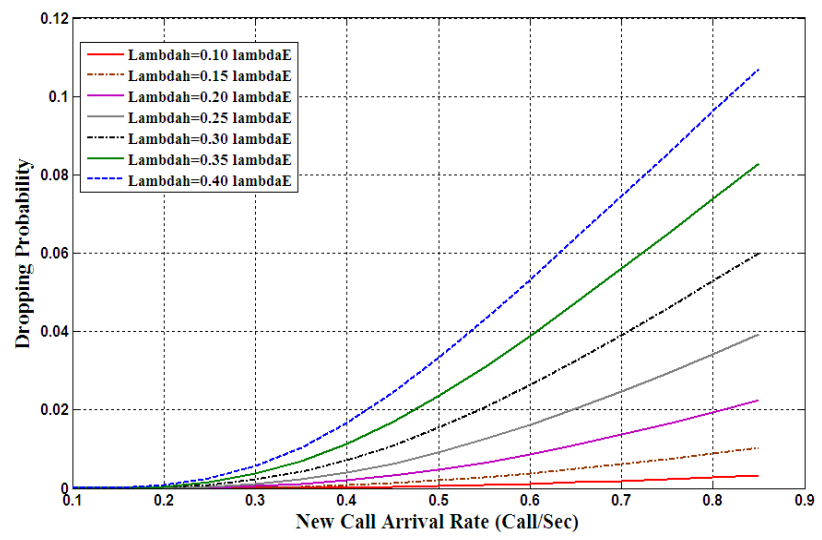

Fig. 4. Dropping probability at different handover arrival rate, $\lambda_{h}, N=48, E=16, E_{e}=12$.

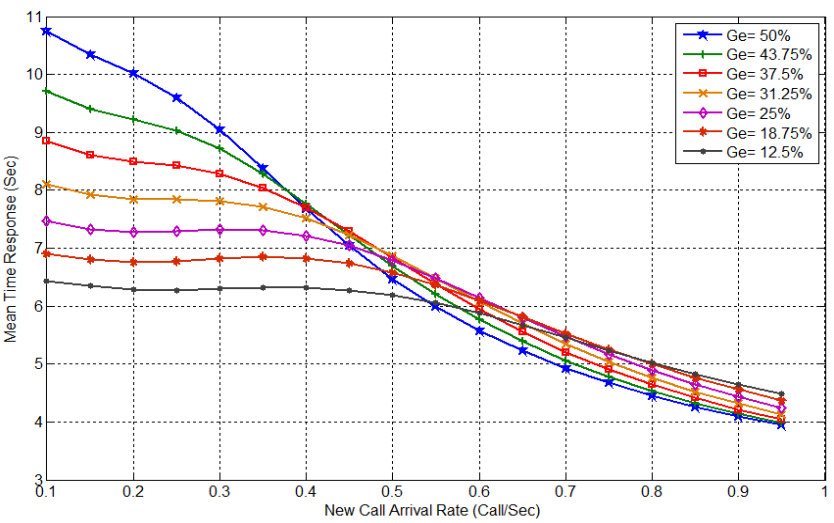

Fig. 6. Average number of users at cell-edge $N=48$, $E=16, E_{e}=12, \lambda_{h}=0.5 \lambda_{e}$ Call $\backslash \mathrm{Sec}, \mu=1 / 90 \mathrm{Sec}-1$. 
When the number of guard channels is large, the remaining RBs decrease which, in turn, increase the mean time response. At heavy traffic, the number of RBs increases by using guard channels which in turn reduces the mean time response.

\section{Conclusion}

In this paper, we discuss analytically the impact of limited fractional guard channel with soft frequency reuse using queuing analysis. From numerical analysis and results we can conclude that the merging of LFGC and SFR models are leading to more inspection of system behavior. The manipulation of LFGC based model or SFR based model cannot be used to analyze the contribution ratio of cell-edge and cell-core users. The obtained results illustrated that the system behaves different according to user location. In addition, the new call arrival rate and number of guard channel have great impact on LFGC blocking probability. Most blocking rate which is caused by handover process occurs at cell-edge. On the other hand, the cell-core users affected, but to a lesser extent, by handover process. Most blocking rate which is caused by handover process occurs at cell-edge. On the other hand, the cell-core users affected by handover process but to a lesser extent. Finally the handover process has noticeable impact in the throughput and mean time response.

\section{References}

[1] Chowdhury, M. Z., Jang, Y. M., \& Haas, Z. J. (2013). Call admission control based on adaptive bandwidth allocation for wireless networks. Journal of Communications and Networks, 15(1), 15-24.

[2] Ramjee, R., Towsley, D., \& Nagarajan, R. (1997). On optimal call admission control in cellular networks. Wireless Networks, 3(1), 29-41.

[3] Vazquez-Avila, J., Cruz-Pérez, F. A., \& Ortigoza-Guerrero, L. (2006). Performance analysis of fractional guard channel policies in mobile cellular networks. IEEE Transactions on Wireless Communications, 5(2), 301-305.

[4] Goswami, V., \& Swain, P. K. (2012). Analysis of finite population limited fractional guard channel call admission scheme in cellular networks. Procedia Engineering, 30, 759-766.

[5] Huawei. (May 2005). R1-050507, soft frequency reuse scheme for UTRAN LTE. Proceedings of Huawei 3GPP TSG RAN WG1 Meeting No. 41. Athens, Greece. form http://www.3gpp.org/ftp/tsg_ran/WG1_RL1/TSGR1_41/Docs/R1-050507.zip

[6] Qian, M., Hardjawana, W., Li, Y., Vucetic, B., Shi, J., \& Yang, X. (April 2012). Inter-cell interference coordination through adaptive soft frequency reuse in LTE networks. Proceedings of the 2012 IEEE Wireless Communications and Networking Conference (pp. 1618-1623).

[7] Korda, L. (July 2001). The making of a translator. Translation Journal, 5(3). from http://accurapid.com/journal/17prof.htm

[8] Shu, L., Wen, X., Liu, Z., Zheng, W., \& Sun, Y. (2010). Queue analysis of soft frequency reuse scheme in LTE-advanced. Proceedings of Second International Conference on Computer Modeling and Simulation, Vol. 1 (pp. 248-252).

[9] Kim, S. Y., Lee, H. W., \& Cho, C. H. (April 2014). Analytical evaluation of call admission control for SFR-based LTE systems. Proceedings of the Tenth International Conference on Networking and Services (pp. 69-73).

[10] Safwat, M. A., El-Badawy, H. M., Yehya, A., \& El-Motaafy, H. (2013). Performance assessment for LTE-advanced networks with uniform fractional guard channel over soft frequency reuse scheme. Wireless Engineering and Technology, 4, 161. 


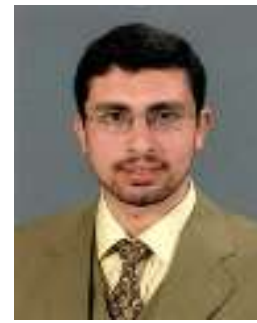

Mahammad A. Safwat received his B.Sc., M.Sc. and Ph.D. degrees in electrical communication engineering from Al-Azhar University, Cairo, Egypt in 2004, 2009 and 2014 respectively. $\mathrm{He}$ is a doctor in the Networks Planning Dept. of National Telecommunication Institute, Ministry of Communication \& Information Technology, Cairo, Egypt. His research interests include networking, performance evaluation techniques, teletraffic modeling, and planning techniques.

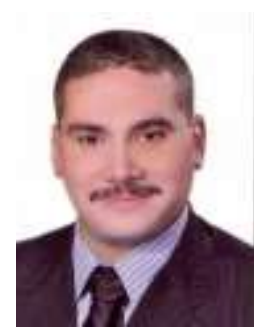

Hesham M. El-Badawy is a full professor and the head of the Networks Planning Dept. of National Telecommunication Institute, Ministry of Communication \& Information Technology, Cairo, Egypt. His research interests include networking, performance evaluation techniques, teletraffic modeling, MIMO systems, and WiMAX networks, ATM networks, digital communication and recently broadband wireless communication systems such as: WCDMA/HSPA, and LTE. He has published more than 70 journal and conference papers and supervised more than 45 Ph.D. and M.Sc. students. Also, he is a recognized instructor in International Telecommunication Union, Prof. Hesham is elevated to be a IEEE senior member since May 2014 in the Vehicular Technology Society as well as the Communications Society.

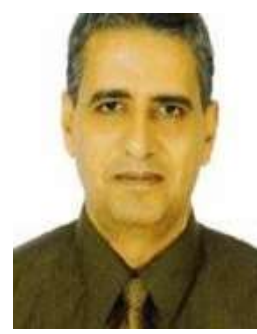

Ahmed Yahya graduated from Ain Shams University and got the Ph.D. degree in 1998 under the supervision of professor Hani Fikry. He was appointed as a lecturer at Alazhar University 1998 and established his research group. His research interest is in the area of computer engineering and computer science at graduate levels. He was promoted to be an associate professor in 2012. 